RESEARCH ARTICLE

\title{
Infection Control and Modified Workflow Strategies in Radiation Oncology Department during COVID 19 Crisis: An Institutional Experience
}

\author{
Sweta Soni, Akanksha Solanki, Puneet Pareek, Rakesh Kumar Vyas, Sumanta \\ Manna, Sanjib Gayen
}

All India Institute of Medical Sciences, Jodhpur, India.

\begin{abstract}
Purpose: To build safe environment for cancer fighters and radiation personnel during COVID-19 pandemic by focusing on infection control, workflow and radiotherapy dose schedules modification strategies in radiation oncology departments. Material and Methods: A meeting was called post lock down in radiation oncology department to prepare infection control policies and workflow strategies in time of COVID-19 Pandemic. Results: Strategies and policies were formed during COVID-19 crises taking following points into consideration 1) Infection control policies 2) CT simulation policies 3) Day care admission and chemotherapy administration policies 4) Radiation treatment plan modification and delivery strategies 5) Brachytherapy delivery strategies. Conclusion: Management of cancer patients is an issue running parallel to the present condition of COVID-19 pandemic. Further randomized trial on hypofractionated radiotherapy schedules should be encouraged. Positivity, awareness and systematic approach are most important step in balancing the current scenario.
\end{abstract}

Keywords: COVID-19- Radiotherapy- Strategy-Infection control- Hypofractionation

Asian Pac J Cancer Care, 6 (Suppl 1), 21-26
Submission Date: 05/22/2021 Acceptance Date: 05/30/2021

\section{Introduction}

COVID-19 pandemic is major health issue since the verge of year 2020. Its first case was reported in December 2019 in Wuhan, a city in China. Now over a period of 4 months the disease have spread to $>150$ countries and the patients have rapidly increased to approximately 30 lakhs, moreover the concern is that it has also led to approximately 2 lakh deaths worldwide. On $12^{\text {th }}$ March, the WHO officially declared that the COVID-19 outbreak as a pandemic caused by SARS-CoV-2 [1]. Various predictions have been made for the spread of COVID-19 including by a leading Harvard epidemiologist Marc Lipsitch who warns that the coronavirus will infect up to 70 per cent of humanity within a year [2]. Moreover, pandemic has startled and created unprecedented pressure on government and health service throughout the world including India.

India being one of the developing country with 1.38 billion population [3] residing in densely populated areas which emerges to be constant risk for outbreaks that can result in rapid spread of infection. In addition, poverty and underdevelopment further intensifies the situation. Apart from general public, COVID-19 pandemic also compromises circumstances for people fighting with cancerous diseases. Cancer patients are predominately immunocompromised, weakened by the burden of the disease and its treatment and other comorbidities and thus exposing them to the severe form of COVID-19 infection. Among 44,000 confirmed cases of COVID-19 in China, $0.9 \%$ of patients who had no underlying medical conditions died, but that number rose to $6 \%$ for people with cancer, reported by the centres for Disease Control and Prevention [4]. More intensive attention should be paid to patients with cancer, in case of rapid deterioration.

Corresponding Author:

Dr. Sweta Soni

All India Institute of Medical Sciences, Jodhpur, India.

Email: sonisweta@hotmail.com 
COVID-19 Pandemic: Predicament for Cancer Patients and Health workers.

The war against COVID-19 is still continuing in India. Currently (As per covid19india.org, dated july $\left.3^{\text {rd }}, 2020\right)$ total number of confirmed cases in India are $6,26,378$, of which 2,28185 are active cases and death toll rising to 18,225 . In Rajasthan total number of COVID-19 confirmed cases reached to 18,662 with active cases being 3,284 and 430 deaths. And presently quarantine and social distancing are only key to prevent COVID-19 outbreak to spread rapidly in community. Cancer and Corona-19 pandemic, presents has double edged situation for cancer fighters. Majority patients diagnosed of malignancy present in advanced stage disease are malnourished with weak innate immunity due to the disease process, poor nutritional status as well as the side effects of therapy (especially chemotherapy). Liang et al.[5] reported a significantly higher incidence of severe events, i.e., death or ICU admission requiring invasive ventilation among individuals with a cancer history than those without ( $39 \%$ vs. $8 \% ; p=0.0003$ ), in 2007 Chinese patients hospitalized with COVID-19. In addition to this large number of patient's finds difficulty in reaching out to hospitals. And radiotherapy being prolonged treatment usually delivered 5 days a week over 4-7 weeks based on cancer site needs attention during such crises. On the other end are health care workers who are constantly at increased risk of exposure for acquiring the infection.

Therefore intention is to create safe environment for cancer fighters and radiation personnel during COVID-19 pandemic by focusing on infection control, workflow strategies and overall functioning of radiation oncology departments.

\section{Materials and Methods}

With announcement of lockdown in India, on $25^{\text {th }}$ March, 2020, a meeting was called in radiation oncology department equipped with one LINAC: Elekta HD Versa and one brachytherapy unit to prepare infection control policies and workflow strategies in time of crises. Meeting included radiation oncologists (Faculty, Senior and Junior Doctors), Medical Physicist, Technologists, Nursing Officers and supporting staff. Major highlights of this discussion was firstly to reduce the risk of transmission amongst inflowing patients and care givers without compromising departmental functioning. Secondly, to modify radiotherapy treatment plans and encouraging use of hypofractionated dose schedules wherever possible.

\section{Results}

The strategies were initially made and applied for patients who were already registered with the hospital and were not coming from containment zone or high risk area. All patients and accompanying person were allowed to enter in department only if they were free from flu symptoms and who have got clearance from Flu screening desk of the hospital.

The infection control and workflow modification strategies was discussed as following: 1) Infection control policies 2) CT simulation policies 3) Day care admission and chemotherapy administration policies 4) Radiation treatment plan modification and delivery strategies 5) Brachytherapy delivery strategies

Infection control plan for Oncology Block

A) Patient waiting area

Preferably, patients are called only after discussion has happened through telemedicine. Prior appointments were given in order to avoid clustering of patients in department. Patients/ relatives with symptoms of cough/ cold/ fever/ breathing difficulty unless strongly associated with malignancy were advised to stay at home for 2 weeks. Any patient with new symptoms of flu was advised to see flu desk closest to their home. Only one relative was allowed with the patient only if necessary. Patients were segregated according to time of radiation therapy or chemotherapy by giving pre-appointments.

B) Patient examination and interaction area

A small curtained area was created at the entry point of department for any routine consultation / looking at documents of patients. Only one patient was allowed at a time as per the appointment given. In case of clustering of patients they were guided to stay out of department in covered area maintaining social distance. An empty room was converted into examination area near to the entry gate. While entering the examination room the doctors were instructed to wear triple layer mask, face shield and gloves. Alcohol based sanitizer was used before and after each patient examination.

C) LINAC and way towards LINAC

We planned to keep patient number to max 3 / hour to use in between time for cleaning and sanitization. Hospital staff were instructed to wipe the couch with either ECOSHIELD (H202+ Silver Nitrate soln. (10 cc in $980 \mathrm{cc}$ water) OR 70\% alcohol (Sanitizers) and leave for $5-7$ minutes after every patient treatment. Every 2 hours during LINAC working, the floor has to be mopped from main gate till LINAC couch including the changing area floor--- with either lyzol or Sodium Hypochlorite [6]. Any tile of wall close to patient like in changing room also has to be wiped with same solution whenever floor is wiped. All technologist were instructed to wear 2 masks preferably --- homemade below and triple layer above. They were instructed not to touch top surface of mask or face once they wear it and if they do so then they were supposed to change the mask by using sanitizer before and after changing it. Also use of sanitizer to clean hands (even above gloves) and treatment console keyboards after and before touching any patient or their cast was advised.

\section{CT simulation policies}

Only 5 patients were called for CT simulation in a week. Of these patients 3 patients were called on Friday and 2 were called on Monday. On the day of simulation the patients due for simulation were asked to get clearance from flu screening desk. After each simulation the couch had to be cleaned with alcohol based sanitizer or Ecoshield. After all simulations the simulation room was 
Table 1. Hypofractioned Radiotherapy Dose Schedules Site-Wise

\begin{tabular}{|c|c|c|c|c|}
\hline NO. & Site & Dose Fractionation & Dose Gy/Fx & Duration \\
\hline 1 & Brain (High Grade) & $40 \mathrm{~Gy} / 15 \mathrm{Fx}$ & 2.67 & 3 weeks \\
\hline \multirow[t]{4}{*}{2} & Head And Neck & Postoperative & & \\
\hline & & $54 \mathrm{~Gy} / 24 \mathrm{Fx}$ & 2.25 & 5 weeks \\
\hline & & Definative & & \\
\hline & & $60 \mathrm{~Gy} / 25 \mathrm{Fx}$ & 2.4 & 5 weeks \\
\hline 3 & Breast & $42.6 \mathrm{~Gy} / 16 \mathrm{Fx}$ & 2.66 & 3 weeks \\
\hline 4 & Cervix & $45 \mathrm{~Gy} / 20 \mathrm{Fx}$ & 2.25 & 4 weeks \\
\hline 5 & Rectum & $45 \mathrm{~Gy} / 20 \mathrm{Fx}$ & 2.25 & 4 weeks \\
\hline 7 & Brachytherapy ( Cervix) & ICBT: 9 Gy X 2 Fx, 1 week apart & 9 & \\
\hline \multirow[t]{9}{*}{8} & Palliative & & & \\
\hline & Brain Mets & $20 \mathrm{~Gy} / 5 \mathrm{Fx}$ & 4 & 5 days \\
\hline & & 15 Gy/ 3 Fx , alternate day & 5 & \\
\hline & & $12 \mathrm{~Gy} / 2$ fraction , 1 week apart & 6 & \\
\hline & Painful Bone Mets & 8 Gy / SINGLE Fx & 8 & single day \\
\hline & Spinal Cord Compression $(<48 \mathrm{Hrs})$ & 8 Gy/ SINGLE Fx & 8 & Single day \\
\hline & Hemostatic Rt & 8 Gy/ SINGLE Fx & 8 & Single day \\
\hline & & $12 \mathrm{~Gy} / 2 \mathrm{Fx}, 1$ week apart & 6 & \\
\hline & SVCO & $20 \mathrm{~Gy} / 5 \mathrm{Fx}$ & 4 & 5 days \\
\hline
\end{tabular}

Fx, Fraction; ICRT, Intracavitaory Brachytherapy; RT, Radiotherapy; SVCO, Superior Venacava Obstruction

instructed to be cleaned with Lyzol/Sodium Hypochlorite. CT simulation was postponed for patient with flu like symptoms. Remaining precautionary measures followed were similar to that of LINAC Area.

\section{Day care admission and chemotherapy administration policies}

Only essential chemotherapies and concurrent chemotherapies were administered in the day care. Maximum patients were advised not to receive intravenous chemotherapy and were shifted on oral chemotherapy (metronomic) regimens via telemedicine consultations. The way to day care in oncology block was cleaned 3 times a day using lyzol. In view to maintain social distance staff was advised to take patients on alternate beds at the time of receiving chemotherapy or any sort of symptomatic treatment. In order to limit the number of patients, morning and evening slots were created and prior appointments were given for chemotherapy using telemedicine system. Each patient had to take clearance from flu screening desk before entering day care. Bed linens were changed after each patient. Preferably only 6 patients (12 beds in total) were treated in each slot. Nursing officers were advised to wear gloves (that are to be changed with proper sanitization after dealing with each patient), triple layer mask and face shield as required. Patients were allowed to enter in day care with proper triple layer masks and hand sanitization (hand washing whenever required). All Aseptic precautions were strictly followed while administrating chemotherapy. Nursing staff were advised to wipe the cannulation area widely with alcohol based sanitized before and after cannulating the patient.

\section{Radiation treatment plan modification and delivery} strategy

We first planned to see list of simulated and registered patients and give priority to following patients in order of registration and priority of early radiation treatment:

- Patients who were diagnosed and those who had received neoadjuvant treatment or underwent surgery at hospital got preference.

- Radical Patients (Definitive CTRT for head and neck malignancies, for cervix, etc) were given higher priority.

- Due to non-functioning of surgical departments the short course NACT-RT regimens for rectum were not preferred, instead patients were started on long course NACT-RT.

- Post Operative Patients -- Head and Neck malignancies who were on verge of completing 100 days since surgery and who had high risk features like positive or close margins, multiple positive neck nodes, etc were given preferences.

- Post operative patients with other malignancies in whom there was limited role of hormonal therapy or adjuvant chemotherapy were given preference.

- Breast Patients for post operative radiation treatment with higher stage and triple negativity were given preference over early stage and hormone positive patients.

- Altered radiation fractionation schedules were used making maximum treatments as hypofractionated wherever possible in order to finish the treatments early. The modified dose fractionation schedules are shown in Table 1.

- Neoadjuvant chemotherapy (intravenous/oral) and neoadjuvant hormonal therapies wherever applicable were started for many patients in March 2020.

- Single fractions were usually planned in palliative 
settings wherever possible. For multiple fractions in palliative settings, fractionations schedule of $20 \mathrm{~Gy} / 5$ Fractions, WBRT 5 Gy x 3 Fractions with treatment every alternate day or 6 Gy $x 2$ Fractions with treatment 1 week to 10 days apart were planned. Single fractions of 6 Gy or 8 Gy were planned for haemostasis.

- Concomitant chemotherapy may be skipped after 3 cycles if tolerance was poor and if patient presented with grade 3 toxicities.

\section{Brachytherapy delivery strategies}

Patients planned for brachytherapy were treated as per schedule after giving a prior appointment. All the patients were advised COVID 19 testing and were considered only when reports were negative. Patients are planned for 9 Gy x 2 Fraction schedule in order to finish the treatment within two weeks.

\section{Discussion}

COVID-19 pandemic is rapidly spreading throughout the world including India, thus jeopardizing the human race and challenging health system of the country. Management of cancer patients is another issue running parallel to the present condition of COVID-19. Mobilizing these patients to come to hospitals for their therapies further increase the chances of their exposure to infection. In oncology patients, risk benefit calculation is very unpredictable. The risk of acquiring COVID-19 infection and at the same time possibility of spread of cancer creates feeling of apprehension amongst cancer fighters. However in present scenario, balancing two situation is the only option. Therefore, strict infection control policies were introduced and workflow /radiotherapy conventional fractionation schemes were modified and hypofractionated treatment protocols were encouraged in order to complete treatment early with tolerable side effects.

\section{Radiotherapy Schedules Modification for various site}

In case of head and neck cancers several alteredfractionation RT regimens have been reported in the literature [7-10]. Jackson et al. [11] observed that 70 patients with $\mathrm{T} 3 \mathrm{~N} 0$ glottic cancer who received a total dose of 60 Gy in twenty-five 2.4 Gy daily fractions over 5 weeks had an initial post radiation primary recurrence free rate of $65 \%$ at 5 years. This dose schedule was minimally less intense in comparison with the protocol of $62.5 \mathrm{~Gy}$ in 25 fractions recently described by Thomson et al. [7] for intermediate-stage HNSCC in a phase 2 trial, albeit with the inclusion of cetuximab as a potential radiosensitizer. Thomson et al. [7] schedule are closer to the more traditional schedule of 2.0 Gy $\times 35$ daily fractions and, therefore, could provide a similar reasonable alternative. Recently published study by Huang et al. [12] observed that hypofractionated radiotherapy (60 Gy/ 25 fraction, 2.4 Gy/ fraction, 5 days a week) may be considered in place of concurrent chemoradiotherapy to $70 \mathrm{~Gy} / 35$ fraction for Human papilloma virus (HPV) positive T1-T3N0-N2c (TNM-7) HNSCCs, HPV- T1-
T2N0 HNSCCs, and select stage III HNSCCs during the COVID-19 outbreak. Most study do not support use of concurrent chemotherapy in hypofractionated setting, but there are few data to support the use of concomitant platinum chemotherapy with higher doses per fraction [13-14]. In present study for definitive chemo-radiotherapy $60 \mathrm{~Gy} / 25$ fraction and in postoperative setting $54 \mathrm{~Gy} / 24$ fraction dose schedule was used.

Arnold et al. [15] observed similar overall survival in high grade glioma patients for hypofractionated arm (40 Gy/ 15 fractions) and Standard radiotherapy arm (60 Gy/30 fractions) with concurrent TMZ among elderly patients. Although further randomized trials are required. In present study high grade gliomas were addressed by $40 \mathrm{~Gy} / 15$ fraction while low grade gliomas were treated with conventional fractionation.

Whelam et al. [16] concluded in his study including breast cancer patients that the risk of local recurrence at 10 years was $6.7 \%$ among the 612 women assigned to standard irradiation (50 Gy/25 fraction) as compared with $6.2 \%$ among the 622 women assigned to the hypofractionated regimen (42.5 Gy/16 fraction) (absolute difference, 0.5 percentage points; $95 \%$ confidence interval [CI], -2.5 to 3.5 ). At 10 years, $71.3 \%$ of women in the control group as compared with $69.8 \%$ of the women in the hypofractionated-radiation group had a good or excellent cosmetic outcome. This hypofractionated regimen (42.5 Gy/16 fractiond) has been used in present study with acceptable outcomes and toxicities. Another study, START-B trial concluded that the proportion of breast cancer patients with local-regional relapse at 10 years did not differ significantly between the $40 \mathrm{~Gy} / 15$ fraction group and the $50 \mathrm{~Gy} / 25$ fraction group $(\mathrm{p}=0 \cdot 21)$. Also breast shrinkage, telangiectasia, and breast oedema were significantly less common normal tissue effects in the 40 Gy group than in the 50 Gy group [17].

In case of cervix carcinoma, study was published from South Africa which included 104 patients with stage IIIB cervical cancers, treated with external beam radiotherapy (EBRT) 40 Gy in 16 daily fractions followed by brachytherapy with 9 Gy $\times 2$ fractions, 1 week apart without concurrent chemotherapy. Complete response was registered in $70 \%$ of patients and disease free-survival (DFS) at 20 months was $59 \%$ and no late geniatourinary toxicities were reported, while 4 late gastrointestinal toxicity were registered [18]. Another, retrospective report from Tata Memorial investigated the role of hypofractionated radiotherapy in 62 stage IIIB cervical cancer patients treated with 39 Gy in 13 daily fractions (mostly AP/PA fields) followed by intracavitary brachytherapy. The 5-year DFS rate was $59 \%$, and 5 patients had late grade 3 rectal toxicity [19]. For brachytherapy, study by Ghosh et al. concluded that 2 year actuarial local tumor control rate, disease-free survival and overall survival was $88.1 \%, 84.2 \%$ and $81.8 \%$ respectively. Grade III late toxicity was seen in only three patients out of 76 . Therefore, 9 Gy / 2 fraction is considered an effective dose fractionation for the treatment of cervical cancer with acceptable toxicity [20]. In present study, advance stage carcinoma cervix patients 
were delivered 45 Gy / 20 fraction, 2.25 Gy/ fraction 5 days a week along with concurrent chemotherapy followed by brachytherapy ( 9 Gy x 2 fractions, 1 week apart) and are kept under follow up for response assessment. There is still a need for further large-scale studies on hypofrac-tionated radiotherapy for cervical cancer.

Myerson et al. [21] concluded that short course radiotherapy 25 Gy $/ 5$ fraction regimen achieved response and morbidity rates that compare favorably with those of conventionally fractionated radiation therapy and concurrent chemotherapy. In present study, short course radiotherapy ( $25 \mathrm{~Gy} / 5$ fraction) was not preferred due to non-functioning of surgical departments initially. Therefore, carcinoma rectum patients were delivered 45 Gy / 20 fraction, $2.25 \mathrm{~Gy} /$ fraction 5 days a week along with concurrent chemotherapy. These patients are considered for long term follow up to assess diseases response and late sequel.

Rich S et al. concluded that in cases with bone metastasis the overall response rate was similar in patients for 8 Gy single fraction treatments $(61 \% ; 1867 / 3059)$ and those for multiple fraction treatments $(62 \% ; 1890 / 3040)$. No significant difference was seen in the risk of pathological fracture at the treatment site, rate of spinal cord compression at the index site, or in the rate of acute toxicity [22].

In case of brain metastasis, study showed that shorter time-dose fractionation schemes such as $30 \mathrm{~Gy} / 10$ fractions or $20 \mathrm{~Gy} / 5$ fraction may be used with equal efficacy [23]. Fractionation schedule delivered in this study are $20 \mathrm{~Gy} / 5$ fraction, $15 \mathrm{~Gy} / 3$ fraction every alternate day and 12 Gy/ 2 fraction 1 week apart. Patients are kept are under strict follow up for response assessment.

Further randomized trials and studies are required to form dedicated hypofractionated radiotherapy protocols not only to deal with present crises but also to reduce prolonged treatment duration in certain sites considering future aspects.

\section{Tips for Patients and Caregivers}

Precautions and awareness are only key to prevent COVID-19 outbreak to spread rapidly. Patients scheduled for radiotherapy should be educated and counselled to wear face mask, maintain adequate social distancing inside and outside hospital, only one attendant should accompany with patient, avoid gatherings, avoid outside food/ drinks during the course of treatment and inform in case of any flu related symptoms. Also patients should be encouraged to keep themselves hydrated and eat healthy and freshly prepared food or in case of ryles tube feeders or with mild to moderate dysphagia should have small frequent feeds.

Care givers should also strictly follow precautionary measures before, during and after delivering treatment and social distancing rule. Department staff duties should be managed and scheduled in shifts. Telemedicine and work from home system should be encouraged. This will help to reduce footfall of patients in department and thus reduce risk of infection spread. Also proper staffing or $50 \%$ staff rule can contribute in effective running of department. Because if any health workers acquires infection or is quarantined, staff working from home can take over and keep department functional. Apart from this, health workers should take care of themselves concentrating on their health and take diet to boost their immunity. In Addition to this stress, anxiety and depression are silent killers which shouldn't be ignored and psychiatric counselling should be taken whenever needed. All these strategies will help to reduce the risk of COVID-19 exposure and transmission amongst patients and health workers. Moreover, patients treated with modified treatment schedules will be considered for long term follow up for assessing the response or any unforeseen treatment related sequel.

In conclusion, COVID-19 pandemic has definitely created pressure on health facilities but it is responsibility of entire medical fraternity to tackle this outbreak with their knowledge and experience without compromising patient's treatment. Hypofractionated radiotherapy schedules should be encouraged in such situations and further studies should be carried out for forming dedicated protocols. In this difficult times, there's saying in sanskrit "Etdapi Gamishayati" which mean This Too, Shall Pass. Therefore, positivity, awareness and systematic approach are most important step in balancing current situation.

\section{References}

1. WHO announces COVID-19 outbreak a pandemic.2020. http:// www.euro.who.int/en/health-topics/health-emergencies/ coronavirus-covid-19/news/news/2020/3/who-announcescovid-19-outbreak-a-pandemic (accessed 16 May 2020).

2. [Internet]. 2020. https://www.en24.news/s/2020/02/40- to70-of-humanity-will-be-infected-says-harvard-scientist (accessed 16 May 2020).

3. Worldpopulationreview.com.2020. https:// worldpopulationreview.com/countries/developingcountries/ (accessed 16 May 2020).

4. Coronavirus is a bigger threat to people with cancer: Advice for patients and survivors [Internet]. TODAY.com.2020. https://www.today.com/health/coronavirus-cancer-risksurvivors-immunocompromised-patients-t175903. (accessed 16 May 2020).

5. Liang W, Guan W, Chen R, Wang W, Li J, Xu K, Li C, Ai Q, Lu W, Liang H, Li S, He J. Cancer patients in SARSCoV-2 infection: a nationwide analysis in China. The Lancet Oncology. 2020 03;21(3):335-337. https://doi.org/10.1016/ s1470-2045(20)30096-6

6. List N: Disinfectants for Use Against SARS-CoV-2 (COVID-19) | US EPA [Internet]. US EPA. 2020 [cited 4 July 2020]. Available from: https://www.epa.gov/pesticideregistration/list-n-disinfectants-use-against-sars-cov-2covid-19.

7. Thomson DJ, Ho KF, Ashcroft L, Denton K, Betts G, Mais KL, Garcez K, Yap BK, Lee LW, Sykes AJ, Rowbottom $\mathrm{CG}$, Slevin NJ. Dose intensified hypofractionated intensitymodulated radiotherapy with synchronous cetuximab for intermediate stage head and neck squamous cell carcinoma. Acta Oncologica. 2014 Oct 03;54(1):88-98. https://doi.org $/ 10.3109 / 0284186 x .2014 .958528$

8. Franzese C, Fogliata A, Franceschini D, Navarria P, Cozzi L, Tomatis S, De Virgilio A, Spriano G, Scorsetti M. Impact of hypofractionated schemes in radiotherapy for locally advanced head and neck cancer patients. The Laryngoscope. 
2020 04;130(4). https://doi.org/10.1002/lary.28048

9. Eisbruch A, Harris J, Garden AS, Chao CK, Straube W, Harari PM, Sanguineti G, Jones CU, Bosch WR, Ang KK. MultiInstitutional Trial of Accelerated Hypofractionated IntensityModulated Radiation Therapy for Early-Stage Oropharyngeal Cancer (RTOG 00-22). International Journal of Radiation Oncology*Biology*Physics. 2010 04;76(5):1333-1338. https://doi.org/10.1016/j.ijrobp.2009.04.011

10. Guckenberger M, Belka C, Bezjak A, Bradley J, Daly ME, DeRuysscher D, Dziadziuszko R, Faivre-Finn C, Flentje M, Gore E, Higgins KA, Iyengar P, Kavanagh BD, Kumar S, Le Pechoux C, Lievens Y, Lindberg K, McDonald F, Ramella S, Rengan R, Ricardi U, Rimner A, Rodrigues GB, Schild SE, Senan S, Simone II CB, Slotman BJ, Stuschke M, Videtic G, Widder J, Yom SS, Palma D. Practice recommendations for lung cancer radiotherapy during the COVID-19 pandemic: An ESTRO-ASTRO consensus statement. Radiotherapy and Oncology. 2020 05;146:223-229. https://doi.org/10.1016/j. radonc.2020.04.001

11. Jackson SM, Hay JH, Flores AD. Local control of T3N0 glottic carcinoma by 60 Gy given over five weeks in 2.4 Gy daily fractions. One more point on the biological effective dose (BED) curve. Radiotherapy and Oncology. 2001 05;59(2):219-220. https://doi.org/10.1016/s01678140(01)00286-9

12. Huang SH, O'Sullivan B, Su J, Ringash J, Bratman SV, Kim J, Hosni A, Bayley A, Cho J, Giuliani M, Hope A, Spreafico A, Hansen AR, Siu LL, Gilbert R, Irish JC, Goldstein D, Almeida J, Tong L, Xu W, Waldron J. Hypofractionated radiotherapy alone with 2.4 Gy per fraction for head and neck cancer during the COVID-19 pandemic: The Princess Margaret experience and proposal. Cancer. 2020 06;126(15):3426-3437. https://doi.org/10.1002/cncr.32968

13. Benghiat H, Sanghera P, Cashmore J, Hodson J, Mehanna H, Simmons R, Massey P, Sangha G, Bode C, Cooper P, Glaholm J, Hartley A. Four Week Hypofractionated Accelerated Intensity Modulated Radiotherapy and Synchronous Carboplatin or Cetuximab in Biologically Staged Oropharyngeal Carcinoma. Cancer and Clinical Oncology. 201406 24;3(2). https://doi.org/10.5539/cco. v3n2p1

14. Jacinto AA, Batalha Filho ES, Viana LDS, De Marchi P, Capuzzo RDC, Gama RR, Boldrini Junior D, Santos CR, Pinto GDJ, Dias JM, Canton HP, Carvalho R, Radicchi LA, Bentzen S, Zubizarreta E, Carvalho AL. Feasibility of concomitant cisplatin with hypofractionated radiotherapy for locally advanced head and neck squamous cell carcinoma. BMC Cancer. 2018 Oct 23;18(1). https://doi.org/10.1186/ s12885-018-4893-5

15. Arvold ND, Tanguturi SK, Aizer AA, Wen PY, Reardon DA, Lee EQ, Nayak L, Christianson LW, Horvath MC, Dunn IF, Golby AJ, Johnson MD, Claus EB, Chiocca EA, Ligon KL, Alexander BM. Hypofractionated Versus Standard Radiation Therapy With or Without Temozolomide for Older Glioblastoma Patients. International Journal of Radiation Oncology*Biology*Physics. 2015 06;92(2):384-389. https:// doi.org/10.1016/j.ijrobp.2015.01.017

16. Whelan TJ, Pignol J, Levine MN, Julian JA, MacKenzie R, Parpia S, Shelley W, Grimard L, Bowen J, Lukka H, Perera F, Fyles A, Schneider K, Gulavita S, Freeman C. LongTerm Results of Hypofractionated Radiation Therapy for Breast Cancer. New England Journal of Medicine. 201002 11;362(6):513-520. https://doi.org/10.1056/nejmoa0906260

17. Haviland JS, Owen JR, Dewar JA, Agrawal RK, Barrett J, Barrett-Lee PJ, Dobbs HJ, Hopwood P, Lawton PA, Magee BJ, Mills J, Simmons S, Sydenham MA, Venables
K, Bliss JM, Yarnold JR. The UK Standardisation of Breast Radiotherapy (START) trials of radiotherapy hypofractionation for treatment of early breast cancer: 10year follow-up results of two randomised controlled trials. The Lancet Oncology. 2013 Oct;14(11):1086-1094. https:// doi.org/10.1016/s1470-2045(13)70386-3

18. Komen A. A retrospective study of advanced carcinoma of the cervix treated with a hypofractionated radiation therapy protocol at the department of radiation oncology. University of the Witwatersrand, Johannesburg, SouthAfrica.

19. Muckaden M. Hypofractionated radiotherapy in carcinoma cervix IIIB: TataMemorial Hospital experience. Indian J Cancer. 2002;9:127-34.

20. Ghosh S, Rao PB, Kotne S. High Dose Rate Brachytherapy in Two 9 Gy Fractions in the Treatment of Locally Advanced Cervical Cancer - a South Indian Institutional Experience. Asian Pacific Journal of Cancer Prevention. 2015 Nov 04;16(16):7167-7170. https://doi.org/10.7314/ apjcp.2015.16.16.7167

21. Myerson RJ, Tan B, Hunt S, Olsen J, Birnbaum E, Fleshman J, Gao F, Hall L, Kodner I, Lockhart AC, Mutch M, Naughton M, Picus J, Rigden C, Safar B, Sorscher S, Suresh R, Wang-Gillam A, Parikh P. Five Fractions of Radiation Therapy Followed by 4 Cycles of FOLFOX Chemotherapy as Preoperative Treatment for Rectal Cancer. International Journal of Radiation Oncology*Biology*Physics. 2014 03;88(4):829-836. https:// doi.org/10.1016/j.ijrobp.2013.12.028

22. Rich SE, Chow R, Raman S, Liang Zeng K, Lutz S, Lam H, Silva MF, Chow E. Corrigendum to "Update of the systematic review of palliative radiation therapy fractionation for bone metastases" [Radiother. Oncol. 126 (2018) 547-557]. Radiotherapy and Oncology. 2019 06;135:201. https://doi. org/10.1016/j.radonc.2019.03.023

23. Borgelt B, Gelber R, Kramer S, Brady LW, Chang CH, Davis LW, Perez CA, Hendrickson FR. The palliation of brain metastases: Final results of the first two studies by the radiation therapy oncology group. International Journal of Radiation Oncology*Biology*Physics. 1980 01;6(1):1-9. https://doi.org/10.1016/0360-3016(80)90195-9

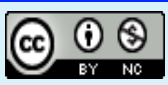

This work is licensed under a Creative Commons AttributionNon Commercial 4.0 International License. 